\title{
Delivery vehicle effects on bone regeneration and heterotopic ossification induced by high dose BMP-2
}

*Laxminarayanan Krishnan, ${ }^{\mathrm{a}}$ *Lauren B Priddy, ${ }^{\mathrm{a}, \mathrm{b}}$ Camden Esancy, ${ }^{\mathrm{a}}$ Brett S Klosterhoff, ${ }^{\mathrm{a}, \mathrm{d}}$ Hazel Y Stevens, ${ }^{\mathrm{d}}$ Lisa Tran, ${ }^{\mathrm{c}}$ and Robert E Guldberg ${ }^{\mathrm{a}, \mathrm{d}}$

*Authors contributed equally to this work.

a. Parker H. Petit Institute for Bioengineering \& Bioscience, Georgia Institute of Technology, 315 Ferst Drive NW, Atlanta, GA 30332, USA

b. Wallace H. Coulter Department of Biomedical Engineering, Georgia Institute of Technology, 313 Ferst Drive NW, Atlanta, GA 30332, USA

c. Department of Surgery, Emory University School of Medicine, Atlanta, GA 30322, USA

d. George W. Woodruff School of Mechanical Engineering, Georgia Institute of Technology, 801 Ferst Drive NW, Atlanta, GA 30332, USA

Address correspondence to:

Robert E. Guldberg, Ph.D.

Parker H. Petit Institute for Bioengineering and Bioscience Georgia Institute of Technology

315 Ferst Drive Atlanta, GA 30332-0363, USA

Email: robert.guldberg@me.gatech.edu

Phone: +1 4048946589

Fax: +1 4048942291

(C) 2016. This manuscript version is made available under the Elsevier user license http://www.elsevier.com/open-access/userlicense/1.0/ 


\begin{abstract}
Bone morphogenetic protein-2 (BMP-2), delivered on absorbable collagen sponge, is frequently used to treat bone defects. However, supraphysiological BMP-2 doses are common and often associated with complications such as heterotopic ossification and inflammation, causing pain and impaired mobility. This has prompted investigations into strategies to spatially control bone regeneration, for example growth factor delivery in appropriate scaffolds. Our objective was to investigate the spatiotemporal effects of high dose BMP-2 on bone regeneration as a function of the delivery vehicle. We hypothesized that an alginate delivery system would spatially restrict bone formation compared to a collagen sponge delivery system. In vitro, BMP2 release was accelerated from collagen sponge compared to alginate constructs. In vivo, bone regeneration was evaluated over 12 weeks in critically sized rat femoral segmental defects treated with $30 \mu \mathrm{g}$ rhBMP-2 in alginate hydrogel or collagen sponge, surrounded by perforated nanofiber meshes. Total bone volume, calculated from micro-CT reconstructions, was higher in the alginate group at 12 weeks. Though bone volume within the central defect region was greater in the alginate group at 8 and 12 weeks, heterotopic bone volume was similar between groups. Likewise, mechanical properties from ex vivo torsional testing were comparable between groups. Histology corroborated these findings and revealed heterotopic mineralization at 2 weeks post-surgery in both groups. Overall, this study recapitulated the heterotopic ossification associated with high dose BMP-2 delivery, and demonstrated that the amount and spatial pattern of bone formation was dependent on the delivery matrix.
\end{abstract}

Keywords - high dose BMP-2, heterotopic ossification, segmental defect Running title - alginate hydrogel delivery of supraphysiological BMP-2 


\section{Introduction}

About $5-10 \%$ of fractures annually in the US suffer from delayed union or non-union, leading to multiple interventions and increased economic burden [1, 2]. Although autograft remains the gold standard in clinical care, failure rates associated with bone grafting interventions range from 13-35\% [3]. BMP-2 has emerged as an effective alternative or adjuvant to autograft tissue or biomaterial scaffolds, based on extensive animal and clinical studies supporting its ability to induce bone formation [4-7]. However, the short half-life of BMP-2 in the blood stream [8,9] and the poor retention of locally delivered BMP-2 [10] have driven scaffold based delivery of BMP-2, with the goal of localized and prolonged bioavailability of BMP-2 at the bone injury site. For example, the absorbable collagen sponge (ACS) scaffold commonly used in the clinic has been shown to retain 30-50\% of the BMP-2 within 3 to 4 days, and $10 \%$ or less within 1 to 2 weeks after delivery [11-14] both at a subcutaneous implant site [12] and in a critically sized femoral segmental defect site [11]. While the disparity between the physiological quantities of BMP in the healing osteogenic environment and the supraphysiological quantities delivered locally is well recognized [15-17], and attributed to differences in activity levels or bioavailability, the optimal spatiotemporal release profile for BMP-2 is yet to be determined.

An optimal sustained delivery profile may promote both chemotactic recruitment of osteoprogenitors to the site of injury and their subsequent differentiation to osteoblasts $[13,18$ 20]. Many parameters could influence whether functional bone regeneration is achieved in response to BMP-2 treatment, including release profile, anatomical and physiological differences, the local vascular and mechanical environments, the use of different scaffolds or adjuvants like bulking agents (TCP/HA: Tricalcium phosphate/ hydroxyapatite, etc.), and of 
course the dose $[21,22]$. While no appreciable differences in healing or adverse effects were noted in a canine spinal fusion model with BMP-2 doses ranging 40-fold (58-2300 $\mu$ g rhBMP-2) delivered in polylactic acid (PLA) carriers [23], many preclinical studies have demonstrated a clear dose effect of BMP delivery. Incremental positive effects of increasing dose of BMP-2 have been observed in a mouse calvarial defect model using heparin conjugated polylactic-coglycolic acid (PLGA) nanospheres and fibrin gel [19], a rat calvarial defect model with PLGA scaffold [24], a rat femoral defect model with both ACS and a hybrid alginate-PCL mesh delivery system [11], a rabbit radial defect model using a PLA scaffold [25], and a canine radial defect model using an ACS scaffold [26]. Moreover, in general, higher doses have been necessary to effectively induce bone formation in non-human primate studies compared to small animal studies [27]. Higher doses of BMP-2 may thus be considered necessary for recruitment or differentiation of progenitor cells for mineralized bridging [28], especially in the absence of bone grafting or bulking agents [22]. However, high dose BMP-2 therapy has also been linked to heightened inflammation [29], production of inflammatory cytokines [30], heterotopic ossification [29, 31, 32], and diminished bone quality [32].

In the clinic, empirical selection of BMP-2 dose may often lead to supraphysiological doses. Given the challenges in clinical therapy of bone non-union, the use of higher doses of rhBMP-2 may provide greater certainty of bone regeneration, and be more acceptable if the side effects of rhBMP-2 excess can be controlled. Despite the known potential complications associated with BMP-2 treatment, few studies have examined different delivery strategies at high doses of BMP-2. Thus, the aim of this study was to provide a direct comparison of the effects of the delivery system on bone regeneration and heterotopic ossification, resulting from a supraphysiological dose of recombinant human BMP-2 (rhBMP-2) - delivered in the clinically 
common ACS or an alginate hydrogel based system. While our group has investigated the hybrid alginate hydrogel system in a pre-clinical animal model of critical sized segmental bone defect [11, 33-41] and demonstrated improved functional bone regeneration compared to autograft [38], and ACS [11, 36, 37] at lower rhBMP-2 doses, the performance of this hybrid system as a carrier for supraphysiological doses of rhBMP-2 has not been evaluated. Moreover,

though exuberant ectopic bone formation has been shown as a part of earlier studies using high dose rhBMP-2, its quantitative characterization and temporal evolution have not been adequately described. This study characterizes the release kinetics of high dose rhBMP-2, bone regeneration, heterotopic ossification, and biomechanical properties of the healed femur. We hypothesized that the alginate delivery system would limit heterotopic ossification, thus providing an improved delivery scaffold for higher doses of BMP-2.

\section{Methods}

\subsection{Delivery vehicle preparation}

A $2 \%$ hydrogel (w/v) of RGD functionalized alginate (FMC BioPolymer, Newark, DE) containing recombinant human bone morphogenetic protein-2 (rhBMP-2, Pfizer Inc., NY) was made as previously described [37, 40]. BMP-2 solutions were formulated in $0.1 \%$ rat serum albumin (RSA, Sigma) in $4 \mathrm{mM} \mathrm{HCl}$ and added in appropriate volumes to yield $30 \mu \mathrm{g}$ of BMP-2 per $150 \mu \mathrm{l}$ hydrogel or media volume. Alginate hydrogels were stored at $4^{\circ} \mathrm{C}$ overnight before use. Sterile biopsy punches $(4 \mathrm{~mm})$ were used to create cylindrical constructs from a $10 \mathrm{~mm}$ thick sheet of sterile collagen sponge (ACS) (Kensey-Nash Corp., Exton, PA). The BMP-2 solution was added drop-wise to the scaffold during surgery and incubated at room temperature for 15 minutes prior to implantation. The therapeutics were delivered in perforated poly $(\varepsilon-$ 
caprolactone) (PCL) nanofiber mesh cylinders (4.5 $\mathrm{mm}$ dia. and $12 \mathrm{~mm}$ length) placed in the bone defect region $[37,40]$. Alginate hydrogel was injected through the perforations in the mesh cylinder while collagen sponge was placed inside the mesh cylinder before loading the BMP-2 solution. Identically prepared delivery vehicles were used for in vitro and in vivo experiments.

\section{2. rhBMP-2 release kinetics}

The in vitro release profile of BMP-2 from three construct groups was assessed: (i) alginate hydrogel surrounded by a mesh, (ii) collagen sponge surrounded by a mesh, and (iii) collagen sponge alone. BMP-2 release was evaluated through 26 days as described previously [40]. Constructs $(\mathrm{n}=6-7)$ containing $30 \mu \mathrm{g}$ BMP-2 were incubated in $1 \mathrm{~mL}$ phosphate buffered saline (PBS), which was collected and replaced at 3 and 6 hours, and at 1, 2, 3, 5, 8, 14, and 26 days. Following the day 26 collection, a vigorous PBS wash was performed to capture residual BMP-2 remaining in the scaffolds. The BMP-2 released in the media at each time point and collected from the terminal PBS wash was quantified using an enzyme-linked immunosorbent assay (ELISA, R\&D Systems, Minneapolis, MN). Cumulative amounts of released BMP-2 were compared over time and among constructs by a two-way repeated measures ANOVA $(\alpha=0.05)$. The apparent retention of BMP-2 was estimated from the measured release over time and expressed as a percentage of the total BMP-2 loaded [40]. BMP-2 release/retention kinetics have previously been represented using exponential decay [11, 40, 42]. Accordingly, BMP-2 retention data for each sample was fit to an exponential decay function, and the rate of decay $\lambda$ was estimated [40].

\subsection{Alkaline phosphatase induction assay}


An alkaline phosphatase (ALP) induction assay using mouse clonal pre-osteoblasts (MC3T3-E1s, American Type Culture Collection, Manassas, VA) was performed to measure the bioactivity of BMP-2 released through 5 days and BMP-2 remaining in the constructs at 26 days $(\mathrm{n}=6-7)$ as previously described $[40,43]$. Mouse clonal pre-osteoblasts (MC3T3-E1, ATCC) were seeded in 96 well plates at a density of 62,500 cells $/ \mathrm{cm}^{2}$ and incubated at $37^{\circ} \mathrm{C}, 5 \% \mathrm{CO}^{2}$ in alpha MEM with 20\% FBS, 1\% penicillin/streptomycin/L-glutamine for 6 hours. Media was then replaced with a 1:1 volume ratio $(200 \mu \mathrm{L}$ total) of: (i) $\boldsymbol{\alpha}$ MEM with $2 \%$ FBS and $0.2 \%$ ascorbic acid 2-phosphate (AA2P); and (ii) PBS containing released BMP-2 collected at 3 hours, 15 hours, and 1, 2, 3, and 5 days — or PBS/SDS containing BMP-2 remaining in the constructs at 26 days. As the assay is completed after 3 days of treatment, little to no ALP activity is seen in the cultures without BMP-2 addition, despite the presence of some ascorbic acid in the media. After 72 hours of culture, MC3T3s were incubated with $7.6 \mathrm{mM}$ p-nitrophenyl phosphate (p-NPP) in $50 \mathrm{mM}$ Tris/ $\mathrm{HCl}(\mathrm{pH} 10.3)$ for $10 \mathrm{~min}$ at $37^{\circ} \mathrm{C}$. The reaction was terminated with $0.2 \mathrm{M} \mathrm{NaOH}$ and the absorbance read on a spectrophotometer $(405 \mathrm{~nm}$, PowerWave X5, Biotek Instruments Inc., Winooski, VT).

\subsection{Surgical procedure}

Unilateral critically sized segmental bone defects were created in the left femora of 13week-old female SASCO Sprague-Dawley rats (Charles River Laboratories, Wilmington, MA) as detailed previously [39]. Briefly, a radiolucent polysulfone plate was first attached to the femur to stabilize it, and an $8 \mathrm{~mm}$ mid-diaphyseal bone segment was excised. Defects were treated with $30 \mu \mathrm{g}$ BMP-2 in collagen sponge or in alginate hydrogel, both surrounded by a PCL nanofiber mesh as described above $(n=9-11)$. Slow release buprenorphine (Wildlife 
Pharmaceuticals, Windsor, CO) was given subcutaneously for analgesia. At the conclusion of the experiments, animals were euthanized by $\mathrm{CO}_{2}$ inhalation. All procedures were approved by the Georgia Institute of Technology Institutional Animal Care and Use Committee (IACUC).

\subsection{Radiography and micro-computed tomography}

Longitudinal bone regeneration was assessed via radiography and micro-computed tomography (micro-CT) through 12 weeks. Radiographs (Faxitron MX-20 Digital, Faxitron Xray Corp., Tucson, AZ) were taken 2, 4, 8, and 12 weeks post-operatively for qualitative observation. De novo mineral ( $\geq 50 \%$ of the density of cortical bone) within and surrounding the defect space was quantified using micro-CT (Viva-CT 40, Scanco Medical, Wayne, PA) at 4, 8, and 12 weeks as established previously. Along the long axis of the femur, the central 136 slices $(\sim 5.3 \mathrm{~mm})$ were evaluated. To differentiate the new bone formation within the bone defect enclosed by the nanofiber mesh from the adjacent heterotopic bone outside the defect, two volumes of interest (VOI) were evaluated. First, a 6-mm diameter VOI was used to characterize mineralization within and immediately bordering the outside of the mesh as evaluated in previous studies using lower BMP-2 doses [40]. Secondly, a large diameter VOI was used to encompass all bone formation within the thigh. The bone volume from the 6-mm VOI (defect bone volume) was subtracted from the bone volume from the corresponding large diameter VOI (total bone volume), and this bone was defined as heterotopic bone. The bone volumes were compared between groups with time using two way repeated measures ANOVA.

Femora were harvested at 12 weeks post-operatively and scanned ex vivo with a voxel size of $21.5 \mu \mathrm{m}$. Here, the large diameter VOI (encompassing both the defect and heterotopic bone) comprised 495 slices ( 10.6 mm of the femur length) and included part of the native bone 
ends. The polar moment of inertia (pMOI), a measure of the bone distribution around the longitudinal (central) axis, was calculated for each slice [35]. All pMOI values in the sample were averaged to provide a global pMOI value for each sample and compared between groups.

\subsection{Biomechanical testing}

The femora harvested at 12 weeks of healing $(n=7-9)$ were tested to failure in torsion (ELF 3200; Bose ElectroForce Systems Group, Eden Prairie, MN) as previously described [39]. Briefly, after clearing of soft tissue and removal of the fixation plate, the bone ends were affixed in Wood's metal (Alfa Aesar, Ward Hill, MA). Maximum torque and torsional stiffness were calculated from torque-rotation curves for each sample and compared between the two groups.

\subsection{Finite element analysis}

Micro-CT based finite element analysis was utilized to estimate the elastic modulus of newly regenerated bone and to decouple the contribution of tissue geometry from experimentally measured torsional stiffness in each sample at 12 weeks. Image-based finite element meshes of the regenerated femora in torsion were created by direct voxel to element conversion of 12 week ex vivo micro-CT scans. Meshes were generated for all defect samples except for one non-union from the alginate treatment group. Each mesh consisted of the entire defect region up to and including approximately $0.2 \mathrm{~mm}$ of intact cortical bone at each end. A suitable element size of $26.25 \mu \mathrm{m}$ was established by a mesh convergence analysis of the torsion model. Typical meshes consisted of approximately 10-16 million first-order hexahedral elements. Intact cortical bone was manually segmented and assigned an isotropic elastic modulus of $10 \mathrm{GPa}$ [44]. Newly regenerated bone was assigned an initial arbitrary homogenized elastic modulus, $E_{F E}$, of 1000 
MPa. The Poisson's ratio of both materials was estimated to be 0.33 [45]. The torsion model boundary conditions prescribed a sub-failure rotation of 0.01 radians about the longitudinal axis of the bone at the proximal end. The distal end was fixed in all directions. The solution to each sample-specific finite element model was utilized to calculate an average tissue level elastic modulus for the newly regenerated bone in each sample by linear correlation with the computed $\left(k_{F E}\right)$ and experimentally measured $\left(k_{\text {Actual }}\right)$ rotational stiffnesses:

$$
E_{\text {Actual }}=E_{F E} \frac{k_{\text {Actual }}}{k_{F E}}
$$

\subsection{Histology}

One sample from each group was harvested at 2 weeks, fixed, and embedded in optimum cutting temperature (OCT) compound. Mid-sagittal $7 \mu$ m non-decalcified sections were obtained from the defect center by a tape transfer technique $[38,46]$ (Section Lab, Hiroshima, Japan). Mineralization was identified by von Kossa staining with Fast Red counterstain. Sequential sections were stained with hematoxylin and eosin $(\mathrm{H} \& \mathrm{E})$ for morphology comparisons. Sections were mounted and imaged on a Zeiss microscope (Axio Observer, Carl Zeiss Microscopy, Thornwood, NY).

One representative sample from each group was also harvested at 4 and 12 weeks and fixed in $10 \%$ neutral buffered formalin. Samples were decalcified, paraffin embedded, and sectioned using a tape transfer technique (Section Labs, Hiroshima, Japan). Sagittal sections obtained from the mid-defect region were deparaffinized and stained with $H \& E$ to characterize tissue morphology, Safranin-O and Fast Green to identify alginate and cartilage, and Mallory's modified aniline blue stain for identification of mature and immature bone/mineralized tissue, as described extensively for this experimental model [11, 36-38, 40]. 


\subsection{Statistical analyses}

Data were analyzed using GraphPad Prism 5 (GraphPad Software, Inc., La Jolla, CA) with a significance level of $\mathrm{p}<0.05$ and reported as mean \pm standard error of the mean $(\mathrm{SEM})$. Release kinetics and longitudinal micro-CT parameters were assessed by two-way repeated measures analysis of variance (ANOVA). BMP-2 recovered from the constructs and decay constants were analyzed by one-way ANOVA or its non-parametric equivalent (Kruskal-Wallis test, followed by Dunn's post-hoc pairwise comparisons). ALP activity of cells cultured with released and retained BMP-2 was evaluated using two-way ANOVA. Post-hoc pairwise comparisons for each ANOVA were performed using the Bonferroni method. Biomechanical properties, including pMOI from micro-CT analyses, were evaluated using Student's t-tests.

\section{Results}

\section{1. rhBMP-2 release kinetics}

The cumulative release of BMP-2 did not differ statistically among the construct groups, though the initial burst release from the collagen sponge group was greater and earlier than that from the alginate. The initial burst release from the collagen sponges, irrespective of the presence of the enveloping mesh, occurred within a day, while that from the alginate occurred between 2 and 5 days (Fig. 1A). Though not significantly different, the presence of the mesh around collagen sponge appeared to retard BMP-2 release after the initial burst. The amount of BMP-2 recovered from the constructs at 26 days was not significantly different among groups (Fig. 1B, Kruskal-Wallis test, $\mathrm{p}=0.16$ ). The BMP-2 retained in the constructs at each time point was calculated from the corresponding rhBMP-2 release data. A non-linear regression analysis 
of this retention data $\left(r^{2}=0.95\right)$ revealed that BMP-2 in the alginate+mesh constructs was released slower than from the collagen+mesh constructs (Fig. 1C). The decay constant from these curve-fits was significantly lower in the alginate+mesh group compared to both collagen groups (Fig 1D; p<0.05, Kruskal-Wallis test), but was comparable to the previously observed decay constant in similar evaluations using alginate hydrogel in a nanofiber mesh with a smaller dose of BMP-2 [40].

\subsection{Alkaline phosphatase induction assay}

Bioactivity of BMP-2 released and retained in the constructs was measured by the ALP assay. ALP activity was normalized to the amount of BMP-2 in the samples at each corresponding time point measured by ELISA. All samples induced measurable ALP activity, with an apparent peak for BMP-2 released at the 1-day time point, but no differences were observed among the groups for released BMP-2 at any time point (Fig. 1E). It must be noted that the ability of the cells to respond to this BMP-2 release may be limited by time or receptor saturation, which may account for the 48-hour window for BMP-2 exposure being sufficient to produce matrix mineralization after 4 weeks in human mesenchymal stem cell cultures [11]. However, the BMP-2 remaining in the alginate+mesh constructs at 26 days induced significantly higher ALP activity compared to BMP-2 retained in the collagen+mesh and collagen constructs (Fig. 1F, p<0.01).

\subsection{Radiography and micro-computed tomography}

The Alginate and Collagen scaffolds in vivo were the same as Alginate+mesh and Collagen + mesh, respectively, from in vitro experiments. Radiographs revealed heterotopic 
ossification beginning at 2 weeks in both groups. Qualitatively, the heterotopic bone was less prominent in both groups by 8 to 12 weeks but appeared more extensive in the collagen sponge group from 4 to 12 weeks (Fig. 2A). More than $90 \%$ of the defects in both groups showed mineralized bridging of the defect by 8 weeks (9/9 of the collagen, and 10/11 of the alginate treated defects). Micro-CT reconstructions at 4, 8, and 12 weeks post-surgery corroborated the radiographic observations. Qualitatively, bone in the collagen group had a highly trabecular structure throughout, both in the defect center and in the heterotopic ossification sites, as seen in the sagittal cross-sections of the bone density maps (Fig. 2B). The alginate treated defects appeared to have more bone in the mesh-bound defect region. At 4 weeks, the alginate group also showed heterotopic bone formation, with defect region mineral increasing with time, in contrast to smaller increases in defect region bone in the collagen treated group.

Quantitative comparison of mineral volume (Fig. 3A-D) showed an increase in total bone volume (defect bone + heterotopic bone) over time for both groups, and total bone volume was significantly greater at 12 weeks in the alginate treated defects than the collagen treated ones $(\mathrm{p}<0.05$, Fig. 3A). Further, bone volume within the defect space was higher for the alginate group at 8 and 12 weeks (p<0.01, Fig. 3B). However, no differences in heterotopic ossification were observed (Fig. 3C). Nonetheless, as a proportion of total bone volume, heterotopic bone volume was significantly attenuated in the alginate group at 8 and 12 weeks ( $<<0.05$, Fig. 3D). At all time points, over $50 \%$ of the total bone volume in the collagen treated defects was heterotopic compared to approximately $30 \%$ of the total bone in the alginate group.

The mineralized trabecular structures both within and surrounding treated defects (total bone volume) were further characterized (Fig. 3E-H). While there were no significant differences between the two groups in the overall number of trabecular structures (Fig. 3E), the 
thickness of these structures was significantly higher in the alginate group at both 8 and 12weeks ( $\mathrm{p}<0.01$, Figs. 3F, 2B). Correspondingly, the trabecular connectivity was reduced significantly in the alginate group at 12 weeks $(\mathrm{p}<0.05$, Fig. 3G), indicating a lower number of connections between the thicker trabeculae. The density of new bone in both groups increased significantly over time but did not differ with the scaffold used (data not shown). The polar moment of inertia (pMOI) for the collagen group had a higher average value but was not significantly different from the alginate group (Fig. 3H).

\subsection{Biomechanical testing and Finite Element Modeling}

No statistically significant differences in the maximum torque at failure or the torsional stiffness between the two groups were noted (Fig. 4A, B). Further, the maximum torque for both groups did not differ from that of intact bone (Fig. 4A) [37]. The average torsional stiffness was significantly higher than historic values reported for intact bone [37, 40], but was not significantly different between the two scaffold groups (Fig. 4B). Sample specific computational modeling was performed to account for the differences in geometry and distribution of mineralized tissue in the defects. Finite element model back-calculation of the elastic tissue modulus (homogenized) of newly regenerated bone demonstrated no differences between the collagen sponge $(778.9 \pm 250.0 \mathrm{MPa})$ and alginate $(813.5 \pm 180.4 \mathrm{MPa})$ treatments at 12 weeks (Fig. 4C). As expected at this stage of healing, the elastic modulus of regenerate bone in both groups was well below that of intact cortical bone $(10-20 \mathrm{GPa})$, but similar to the reported properties of newly formed woven bone [35, 47, 48]. The model strain distribution at a subfailure rotation identified large strains in the outermost connected regions (of heterotopic bone) 
and minimal strain along the central axis in both groups, demonstrating central bone contributes relatively little to the mechanical properties measured by torsion testing (Fig. 4D).

\subsection{Histology}

Non-decalcified cryosections stained with hematoxylin and eosin revealed early mineralization in some areas at the defect edges and more importantly many nodules with cell morphology similar to that of chondrocytes in the surrounding soft tissue in both scaffold groups (Fig. 5A, C). The spatial extent and appearance of heterotopic ossification was similar in both groups. von Kossa staining confirmed mineralization of the defect regions and the surrounding heterotopic nodules (Fig. 5B, D). Mineral deposition was evident in the peri-cellular regions around cartilage-like cells in the nodules in both groups.

Representative paraffin sections captured the developing mineralization over time from 4 to 12 weeks of healing (Fig. 6-7). At 4 weeks (Fig. 6), the collagen treated group contained trabecular structures interspersed with abundant marrow like space with cells in the defect center (Fig. 6A, H\&E stain) and occasional cartilage regions were seen (Fig. 6B, bright red areas with Safranin-O stain). Most bone in the defect appeared to be mature bone, based on the dark red staining with Mallory's modified aniline blue stain (Fig 6C) as described previously [38]. In contrast, the alginate group showed areas of bone interrupted by large pieces of alginate (Fig. 6D-E). Areas of relatively mature bone (orange-red, Fig. 6F) and loose connective tissue were also noted. In both groups, the heterotopic bone had a similar appearance, appeared mature, and was closely associated with the surrounding soft tissue (Fig. 6G-L).

By 12 weeks, the appearance of spongy bone with thin, mature trabeculae was unmistakable within the defects in the collagen treated group with no cartilage detected at this 
stage (Fig. 7A-C). A large part of the section appeared to be loose connective tissue with marrow. In sharp contrast, the alginate group showed more mineral tissue with some marrowlike areas interspersed (Fig. 7D) as well as several smaller persisting remnants of alginate (Fig. 7E) adjacent to mature bone (Fig. 7F) within the defect. The morphology of the heterotopic bone did not appear to change significantly between 4 and 12 weeks in either group. As noted in the micro-CT reconstructions, the collagen treated defects showed a similar amount of mineral within the defect as in surrounding heterotopic sites (Fig. 7G). Compared to the collagen group, the alginate treated defects showed a comparable extent of heterotopic ossification into the surrounding soft tissues, but a higher amount of mineral was noted in the defect center (Fig. 7H).

\section{Discussion}

Pre-clinical animal models and clinical evidence have established BMP-2 as a therapeutic intervention in bone regeneration, but there are conflicting reports on its efficacy, at least in spinal fusion and tibial non-unions [50-53]. It is conceivable that both therapeutic efficacy and the incidence of adverse effects may be dictated by dose and delivery approaches, in addition to other factors. Further, the dose-response relationship of BMP-2 to functional bone regeneration, especially in large bone defects or with higher BMP-2 doses, remains unclear. This study characterized the spatiotemporal differences in bone regeneration and heterotopic ossification in segmental defects and quantified the functional biomechanical properties of the healed defects. The goal was to understand the role of the delivery scaffolds with respect to the observed spatial pattern of bone formation and functional recovery of large bone defects treated with high dose BMP-2. As hypothesized, delivery strategy altered the spatial pattern of bone formation in response to high dose BMP delivery, with the alginate system inducing greater bone formation 
within the defect region. However, contrary to our expectations, the alginate system with less burst release than collagen did not effectively reduce heterotopic ossification.

Clinical use of off-label and high dose BMP-2 is common, and may be associated with adverse effects [51]. The high incidence of side effects may be attributable to the use of supraphysiological doses of BMP-2 and poor retention of BMP-2 by scaffolds. Better BMP-2 delivery and presentation strategies are actively pursued to guide bone regeneration $[54,55]$. Here we evaluated the influence of two delivery scaffolds loaded with the same BMP-2 dose of $120 \mu \mathrm{g} / \mathrm{kg}$ (30 $\mu \mathrm{g}$ per average $250 \mathrm{~g}$ rat), which falls in the typical range of doses used clinically: $50-800 \mu \mathrm{g} / \mathrm{kg}$ (based on the assumption of an $80 \mathrm{~kg}$ individual) [51, 56]. More importantly, previous studies in this rat segmental defect model $[40,57,58]$ have established $2-2.5 \mu \mathrm{g}$ BMP2 as the healing dose (12-15 times lower than the dose used here), with superior retention and healing in alginate scaffolds compared to ACS [11] and autograft (5 $\mu \mathrm{g}$ BMP-2 dose) [38].

In this study, significant differences were seen between the BMP-2 release profiles from the collagen and alginate scaffolds in vitro, which were hypothesized to influence both heterotopic and defect bone formation. However, the slower release from the alginate group appeared insufficient to reduce the heterotopic bone formation. Equivalent heterotopic bone formation was seen as early as 2-4 weeks after surgery in both scaffold groups and could be attributed to the release of a large proportion (>25\%) of the delivered BMP-2 within the first 5 days from both delivery systems. Increased calcium deposition noted in human mesenchymal stem cells 4 weeks after exposure to $100 \mathrm{ng} / \mathrm{ml}$ BMP- 2 for 48 hours supports this conclusion [11]. At 26 days in vitro, alginate hydrogel scaffolds showed higher average retention of the BMP-2 and higher bioactivity. In vivo, this phenomenon may have contributed to the spatial differences in bone formation. Micro-CT data showed that the alginate delivery system 
facilitated higher bone deposition in the central defect region. Approximately $75 \%$ of the total bone in the alginate group was centrally located at 8 and 12 weeks, compared to less than $50 \%$ for the collagen group. This may be due to delayed scaffold disintegration, as inferred from alginate remaining in the defects at 12 weeks in this study and previously $[38,40]$, and increased retention of BMP-2 in the alginate treated group. Though not directly quantified here, the presumed early collapse of the local chemotactic gradient for collagen sponge, as inferred from the high decay constant, and the lower amount of BMP-2 recovered in vitro at day 26 , may explain the differences in bone formation patterns between the two delivery systems.

The structure-function relationship of regenerated bone was investigated to identify the impact of mineral distribution on mechanical properties. Thicker trabeculae with lower connectivity, and heterotopic ossification around the defect region, observed in the alginate group from micro-CT quantifications were in agreement with the histological appearance of the healing defects, where the central defect region showed higher mineralization. For collagen scaffolds, the regenerated bone had a trabecular appearance with large marrow and fibrous tissue infiltrate that has been previously described as abnormal cystic structures [32]. It must however be pointed out that trabecular appearance of regenerated bone was also previously noted in collagen sponges with the use of smaller $(5 \mu \mathrm{g})$ BMP-2 doses [36]. The total bone volumes in this study were comparable to those seen with lower doses of BMP-2 in alginate hydrogel [40]. The lack of significant differences between the two scaffolds in their functional biomechanical properties despite higher defect bone formation in alginate group could be related to the extent of heterotopic bone formation both in its volume and spatial extent (pMOI) for both scaffolds. The torsional stiffness was comparable to that reported for lower doses of BMP-2 in alginate scaffolds [40], indicating limited advantages (if any) in the use of high BMP-2 doses routinely. 
To isolate the tissue-level elastic modulus, an indicator of functional bone maturation, from the torsional stiffness, a sample-specific finite element analysis was used to back-calculate the homogenized modulus from experimental torsion test data of each healed defect. The lack of differences between groups indicated that functional bone maturation at 12 weeks was similar in both the alginate and collagen sponge treatments. The presence of equivalent amounts of histologically mature heterotopic bone in the two groups may account for the lack of differences in homogenized modulus, at least in the torsional test configuration, despite differences in central (defect) bone formation.

Highly mineralized cartilage tissue, suggestive of endochondral ossification, was apparent in the soft tissue surrounding the defects as early as 2 weeks after surgery, as was reported previously in relation to increasing doses of BMP-2 in a subcutaneous implant model [59]. However, higher defect bone formation in the alginate group over time may have resulted from a more sustained chemotactic gradient for progenitor cells that promoted a reparative

response. The initial quantitative characterization of bone regeneration in segmental defects treated with supraphysiological dose of rhBMP-2 in alginate and collagen delivery systems provides the basis to investigate, in later studies, the accompanying inflammation, and its relationship to mineralization, as well as biomaterial therapeutics that can limit heterotopic ossification.

\section{Conclusions}

The alginate hydrogel, with greater BMP retention, augmented BMP-2 induced bone regeneration within the defect boundary, as compared to the loose trabecular bone formation in the defect space seen with collagen based delivery of higher BMP-2 doses, but did not prevent 
heterotopic ossification. The stark differences in amount and distribution of de novo bone observed in this study suggest that the alginate delivery system may be superior to the collagen sponge when using high dose BMP-2, at least in terms of better spatial localization of bone to the defect region. Though saturation limits for such growth factor binding and optimal release kinetics may exist, this study clearly demonstrates that acute exposure to high levels of BMP-2 may be sufficient to induce heterotopic bone formation. There is thus a continued need to identify optimal delivery systems that provide an effective chemotactic gradient, improve bone healing in the region of injury, and limit heterotopic ossification.

\section{Acknowledgements}

This work was supported by the Army, Navy, NIH, Air Force, VA, and Health Affairs to support the AFIRM II effort, under Award No. W81XWH-14-2-0003. Opinions, interpretations, conclusions, and recommendations are those of the authors and are not necessarily endorsed by the Department of Defense. This work was also supported by NIH T32EB006343. Funding sources had no direct involvement in these studies except regulatory oversight. rhBMP-2 (Pfizer, Inc.) and collagen sponge (Kensey-Nash Corp.) were received as gifts. The authors acknowledge Albert Cheng, Chelsea Fechter, Marian Hettiaratchi, David Reece, Sanjay Sridaran, Brennan Torstrick, Jason Wang, Dr. Ashley Allen, Dr. Brent Uhrig, and Dr. Nick Willett for assistance with surgeries, Dr. Laura O'Farrell for veterinary assistance, and Dr. Matthew Priddy for regression analyses. 


\section{Figure Legends}

Figure 1. Release kinetics and bioactivity of BMP-2 from constructs in vitro. (A) Cumulative BMP-2 release through 26 days indicated delayed release through day 5 from alginate+mesh constructs. (B) Minimal BMP-2 was measured in the constructs at 26 days. (C) Plots of BMP-2 retained in the constructs over time alongside the average exponential curve fits (denoted by fit). (D) From curve fits in (C), the decay constant $\lambda$ was significantly higher for the collagen+mesh constructs $(* * \mathrm{p}<0.01)$ and collagen constructs $(* \mathrm{p}<0.05)$ compared to alginate+mesh constructs. (E) ALP activity induced in MC3T3 cells incubated with the sample solutions collected at 3 hours through 5 days, containing the released BMP-2 did not differ significantly among groups. (F) However, significantly higher ALP activity (normalized to amount of BMP-2) was induced by BMP-2 retained (at 26 days) in alginate+mesh constructs compared to that retained in both collagen delivery systems $(* * p<0.01)$. Note: The Alginate+mesh and Collagen + mesh scaffolds here are the same as Alginate and Collagen, respectively, from in vivo experiments.

Figure 2. Longitudinal radiographs and micro-CT reconstructions of regenerating bone defects.

(A) Radiographs showed heterotopic ossification outside the defect site by 2 weeks in both groups, which became less pronounced over time, especially in the alginate group. (B) MicroCT image reconstructions and sagittal cross-sections showed a similar pattern and density of mineral in both groups.

Figure 3. Regenerated bone volumes and morphological parameters over 12 weeks. (A) Total bone volume (BV) increased over time and was significantly higher in the alginate group at 12 
weeks (*p<0.05). (B) Bone volume within the defect region (defect BV) was significantly greater in the alginate group at 8 and 12 weeks $(* * \mathrm{p}<0.01, * * * \mathrm{p}<0.001)$. (C) No differences in the amount of heterotopic bone were observed. (D) Nonetheless, as a proportion of total bone volume, heterotopic bone was significantly lower for the alginate group at 8 and 12 weeks $\left({ }^{*} \mathrm{p}<0.05\right)$. (E) The overall number of bone trabecular structures present was not significantly different between groups. (F) Trabecular thickness was significantly increased at 8 and 12 weeks in the alginate group $\left(* * \mathrm{p}<0.01,{ }^{* * *} \mathrm{p}<0.001\right)$. (G) Correspondingly, connectivity density, a representation of the number of connections per unit volume, was higher in the collagen treated defects at 12 weeks $\left({ }^{*} \mathrm{p}<0.05\right)$. (H) Average polar moment of inertia (pMOI) evaluated from micro-CT reconstructions of 12-week samples was not significantly different between groups.

Figure 4. Biomechanical properties of regenerated bone defect tissue. From torsional testing of bone defect tissue at 12 weeks, no differences in maximum torque to failure (A) or torsional stiffness (B) were observed. However, torsional stiffness values were significantly greater than those of intact bone (dashed lines indicate mean values for historical intact control bone [37], $\left.{ }^{*} \mathrm{p}<0.05\right)$. (C) Homogenized elastic modulus calculated from the sample specific finite element modeling. (D) Representative FE mesh models for each group and a map of equivalent strain experienced by different regions of the samples. No effect of regenerate bone geometry was apparent from the FE modeling based on the strain distribution map $(0-8000 \mu$ strain $)$ and the homogenized modulus.

Figure 5. Routine histology at 2-weeks (cryosections). Many mineral nodules were identified in

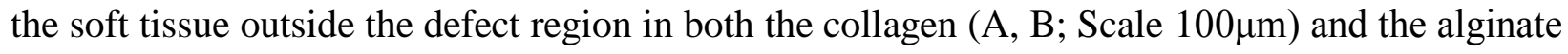


(C, D; Scale $100 \mu \mathrm{m})$ treated groups. Abundant cells with large nuclei and morphology resembling chondrocytes were present. Significant mineral deposition was observed in the pericellular regions within these nodules by von Kossa staining (B, D).

Figure 6. Bone regeneration at 4 weeks. Decalcified and paraffin processed sections were stained with H\&E, Safranin-O, and Mallory's aniline blue. Within the defect, the collagen treated group contained trabecular structures interspersed with abundant marrow like space containing cells and loose fibrous tissue in the defect center (A, H\&E), and occasional cartilage regions were seen (B, bright red, with cells, Safranin-O). Most bone in the defect appeared to be mature (C, red, Mallory's). In contrast, the alginate group (D-F) showed areas of bone and marrow like structures interrupted by large pieces of alginate (E, red, acellular), with areas of relatively mature bone (F, orange-red). In both groups, the heterotopic bone was closely associated with the surrounding soft tissue and had a similar appearance $(G, J)$, no regions of cartilage formation $(\mathrm{H}, \mathrm{K})$, and appeared mature (I, L). Scale: $500 \mu \mathrm{m}$.

Figure 7. Bone regeneration at 12 weeks. Decalcified and paraffin processed sections were stained with H\&E, Safranin-O, and Mallory's aniline blue. (A-C) Thin trabecular structures with loose fibrous tissue were noted within the defects in the collagen treated group with no cartilage (B), and mostly mature bone (C) detected at this stage. In sharp contrast, the alginate group showed more bone areas with some marrow-like tissue interspersed (D), as well as several smaller persisting remnants of alginate (E) adjacent to mature bone $(\mathrm{F})$ within the defect. Lower magnification $(4 \mathrm{x}, \mathrm{H} \& \mathrm{E}$, Scale $2 \mathrm{~mm})$ images revealed that the defect space and surrounding heterotopic sites $(*)$ in collagen treated defects contained similar amounts of mineral $(\mathrm{G})$. 
However, the alginate treated defects showed more bone formation in the defect region, despite having a comparable amount of heterotopic ossification $(*)$ in the surrounding soft tissues $(\mathrm{H})$. Scale: $500 \mu \mathrm{m}(\mathrm{A}-\mathrm{F}), 2 \mathrm{~mm}(\mathrm{G}, \mathrm{H})$. 


\section{References}

[1] N.K. Kanakaris, P.V. Giannoudis, The health economics of the treatment of long-bone nonunions, Injury 38 Suppl 2 (2007) S77-84.

[2] I. Pountos, T. Georgouli, S. Pneumaticos, P.V. Giannoudis, Fracture non-union: Can biomarkers predict outcome?, Injury 44(12) (2013) 1725-32.

[3] M. Geiger, R.H. Li, W. Friess, Collagen sponges for bone regeneration with rhBMP-2, Advanced drug delivery reviews 55(12) (2003) 1613-29.

[4] K.S. Cahill, J.H. Chi, A. Day, E.B. Claus, Prevalence, complications, and hospital charges associated with use of bone-morphogenetic proteins in spinal fusion procedures, Jama 302(1) (2009) 58-66.

[5] J.R. Lieberman, A. Daluiski, T.A. Einhorn, The role of growth factors in the repair of bone, J Bone Joint Surg 84(6) (2002) 1032-1044.

[6] A. Nauth, B. Ristevski, R. Li, E.H. Schemitsch, Growth factors and bone regeneration: how much bone can we expect?, Injury 42(6) (2011) 574-9.

[7] M.F. Swiontkowski, H.T. Aro, S. Donell, J.L. Esterhai, J. Goulet, A. Jones, P.J. Kregor, L. Nordsletten, G. Paiement, A. Patel, Recombinant human bone morphogenetic protein-2 in open tibial fractures. A subgroup analysis of data combined from two prospective randomized studies, The Journal of bone and joint surgery. American volume 88(6) (2006) 1258-65.

[8] Summary of Safety and Effectiveness Data (SSED) for INFUSE Bone Graft, Food and Drug Administration, 2004, pp. 1-29.

[9] F. Liu, P. Misra, E.P. Lunsford, J.T. Vannah, Y. Liu, R.E. Lenkinski, J.V. Frangioni, A doseand time-controllable syngeneic animal model of breast cancer microcalcification, Breast cancer research and treatment 122(1) (2010) 87-94.

[10] P.Q. Ruhe, O.C. Boerman, F.G. Russel, A.G. Mikos, P.H. Spauwen, J.A. Jansen, In vivo release of rhBMP-2 loaded porous calcium phosphate cement pretreated with albumin, Journal of materials science. Materials in medicine 17(10) (2006) 919-27.

[11] J.D. Boerckel, Y.M. Kolambkar, K.M. Dupont, B.A. Uhrig, E.A. Phelps, H.Y. Stevens, A.J. Garcia, R.E. Guldberg, Effects of protein dose and delivery system on BMP-mediated bone regeneration, Biomaterials 32(22) (2011) 5241-51.

[12] W. Friess, H. Uludag, S. Foskett, R. Biron, C. Sargeant, Characterization of absorbable collagen sponges as recombinant human bone morphogenetic protein-2 carriers, International journal of pharmaceutics 185(1) (1999) 51-60.

[13] R.H. Li, J.M. Wozney, Delivering on the promise of bone morphogenetic proteins, Trends in biotechnology 19(7) (2001) 255-65.

[14] H. Uludag, T. Gao, T.J. Porter, W. Friess, J.M. Wozney, Delivery systems for BMPs: factors contributing to protein retention at an application site, The Journal of bone and joint surgery. American volume 83-A Suppl 1(Pt 2) (2001) S128-35.

[15] E.H.J. Groeneveld, E.H. Burger, Bone morphogenetic proteins in human bone regeneration, Eur J Endocrinol 142(1) (2000) 9-21.

[16] R.F. Service, Tissue engineers build new bone, Science 289(5484) (2000) 1498-500.

[17] E.A. Wang, V. Rosen, P. Cordes, R.M. Hewick, M.J. Kriz, D.P. Luxenberg, B.S. Sibley, J.M. Wozney, Purification and Characterization of Other Distinct Bone-Inducing Factors, P Natl Acad Sci USA 85(24) (1988) 9484-9488. 
[18] K.V. Brown, B. Li, T. Guda, D.S. Perrien, S.A. Guelcher, J.C. Wenke, Improving bone formation in a rat femur segmental defect by controlling bone morphogenetic protein- 2 release, Tissue engineering. Part A 17(13-14) (2011) 1735-46.

[19] W.G. La, S.W. Kang, H.S. Yang, S.H. Bhang, S.H. Lee, J.H. Park, B.S. Kim, The efficacy of bone morphogenetic protein-2 depends on its mode of delivery, Artificial organs 34(12) (2010) 1150-3.

[20] O. Jeon, S.J. Song, H.S. Yang, S.H. Bhang, S.W. Kang, M.A. Sung, J.H. Lee, B.S. Kim, Long-term delivery enhances in vivo osteogenic efficacy of bone morphogenetic protein-2 compared to short-term delivery, Biochemical and biophysical research communications 369(2) (2008) 774-80.

[21] O. Arosarena, W. Collins, Comparison of BMP-2 and -4 for rat mandibular bone regeneration at various doses, Orthodontics \& craniofacial research 8(4) (2005) 267-76.

[22] D.S. Mulconrey, K.H. Bridwell, J. Flynn, G.A. Cronen, P.S. Rose, Bone morphogenetic protein (RhBMP-2) as a substitute for iliac crest bone graft in multilevel adult spinal deformity surgery: minimum two-year evaluation of fusion, Spine 33(20) (2008) 2153-9.

[23] H.S. Sandhu, L.E. Kanim, J.M. Kabo, J.M. Toth, E.N. Zeegen, D. Liu, R.B. Delamarter, E.G. Dawson, Effective doses of recombinant human bone morphogenetic protein-2 in experimental spinal fusion, Spine 21(18) (1996) 2115-22.

[24] C.M. Cowan, T. Aghaloo, Y.F. Chou, B. Walder, X. Zhang, C. Soo, K. Ting, B. Wu, MicroCT evaluation of three-dimensional mineralization in response to BMP-2 doses in vitro and in critical sized rat calvarial defects, Tissue engineering 13(3) (2007) 501-12.

[25] H.D. Zegzula, D.C. Buck, J. Brekke, J.M. Wozney, J.O. Hollinger, Bone formation with use of rhBMP-2 (recombinant human bone morphogenetic protein-2), The Journal of bone and joint surgery. American volume 79(12) (1997) 1778-90.

[26] M.F. Sciadini, K.D. Johnson, Evaluation of recombinant human bone morphogenetic protein-2 as a bone-graft substitute in a canine segmental defect model, Journal of orthopaedic research : official publication of the Orthopaedic Research Society 18(2) (2000) 289-302.

[27] G.J. Martin, Jr., S.D. Boden, M.A. Marone, M.A. Marone, P.A. Moskovitz, Posterolateral intertransverse process spinal arthrodesis with rhBMP-2 in a nonhuman primate: important lessons learned regarding dose, carrier, and safety, Journal of spinal disorders 12(3) (1999) 17986.

[28] H. Seeherman, The influence of delivery vehicles and their properties on the repair of segmental defects and fractures with osteogenic factors, The Journal of bone and joint surgery. American volume 83-A Suppl 1(Pt 2) (2001) S79-81.

[29] L.B. Shields, G.H. Raque, S.D. Glassman, M. Campbell, T. Vitaz, J. Harpring, C.B. Shields, Adverse effects associated with high-dose recombinant human bone morphogenetic protein-2 use in anterior cervical spine fusion, Spine 31(5) (2006) 542-7.

[30] K.B. Lee, C.E. Taghavi, K.J. Song, C. Sintuu, J.H. Yoo, G. Keorochana, S.T. Tzeng, Z. Fei, J.C. Liao, J.C. Wang, Inflammatory characteristics of rhBMP-2 in vitro and in an in vivo rodent model, Spine 36(3) (2011) E149-54.

[31] H. Deutsch, High-dose bone morphogenetic protein-induced ectopic abdomen bone growth, The spine journal : official journal of the North American Spine Society 10(2) (2010) e1-4.

[32] J.N. Zara, R.K. Siu, X. Zhang, J. Shen, R. Ngo, M. Lee, W. Li, M. Chiang, J. Chung, J. Kwak, B.M. Wu, K. Ting, C. Soo, High doses of bone morphogenetic protein 2 induce structurally abnormal bone and inflammation in vivo, Tissue engineering. Part A 17(9-10) (2011) 1389-99. 
[33] E. Alsberg, H.J. Kong, Y. Hirano, M.K. Smith, A. Albeiruti, D.J. Mooney, Regulating Bone Formation via Controlled Scaffold Degradation, Journal of Dental Research 82(11) (2003) 903908.

[34] J.D. Boerckel, K.M. Dupont, Y.M. Kolambkar, A.S. Lin, R.E. Guldberg, In vivo model for evaluating the effects of mechanical stimulation on tissue-engineered bone repair, J Biomech Eng 131(8) (2009) 084502.

[35] J.D. Boerckel, Y.M. Kolambkar, H.Y. Stevens, A.S. Lin, K.M. Dupont, R.E. Guldberg, Effects of in vivo mechanical loading on large bone defect regeneration, Journal of orthopaedic research : official publication of the Orthopaedic Research Society 30(7) (2012) 1067-75.

[36] Y.M. Kolambkar, J.D. Boerckel, K.M. Dupont, M. Bajin, N. Huebsch, D.J. Mooney, D.W. Hutmacher, R.E. Guldberg, Spatiotemporal delivery of bone morphogenetic protein enhances functional repair of segmental bone defects, Bone 49(3) (2011) 485-92.

[37] Y.M. Kolambkar, K.M. Dupont, J.D. Boerckel, N. Huebsch, D.J. Mooney, D.W. Hutmacher, R.E. Guldberg, An alginate-based hybrid system for growth factor delivery in the functional repair of large bone defects, Biomaterials 32(1) (2011) 65-74.

[38] L. Krishnan, L.B. Priddy, C. Esancy, M.T. Li, H.Y. Stevens, X. Jiang, L. Tran, D.W. Rowe, R.E. Guldberg, Hydrogel-based Delivery of rhBMP-2 Improves Healing of Large Bone Defects Compared With Autograft, Clinical orthopaedics and related research (2015).

[39] M.E. Oest, K.M. Dupont, H.J. Kong, D.J. Mooney, R.E. Guldberg, Quantitative assessment of scaffold and growth factor-mediated repair of critically sized bone defects, Journal of orthopaedic research : official publication of the Orthopaedic Research Society 25(7) (2007) 941-50.

[40] L.B. Priddy, O. Chaudhuri, H.Y. Stevens, L. Krishnan, B.A. Uhrig, N.J. Willett, R.E. Guldberg, Oxidized alginate hydrogels for bone morphogenetic protein-2 delivery in long bone defects, Acta biomaterialia 10(10) (2014) 4390-9.

[41] C.A. Simmons, E. Alsberg, S. Hsiong, W.J. Kim, D.J. Mooney, Dual growth factor delivery and controlled scaffold degradation enhance in vivo bone formation by transplanted bone marrow stromal cells, Bone 35(2) (2004) 562-569.

[42] F.O. Ribeiro, M.J. Gomez-Benito, J. Folgado, P.R. Fernandes, J.M. Garcia-Aznar, In silico Mechano-Chemical Model of Bone Healing for the Regeneration of Critical Defects: The Effect of BMP-2, PloS one 10(6) (2015) e0127722.

[43] M. Wiemann, H.M. Rumpf, D. Bingmann, H.P. Jennissen, The Binding of rhBMP-2 to the Receptors of viable MC3T3-E1 Cells and the Question of Cooperativity, Materialwissenschaft und Werkstofftechnik 32(12) (2001) 931-936.

[44] H. Oxlund, N.B. Andersen, G. Ortoft, H. Orskov, T.T. Andreassen, Growth hormone and mild exercise in combination markedly enhance cortical bone formation and strength in old rats, Endocrinology 139(4) (1998) 1899-904.

[45] D. Ulrich, B. van Rietbergen, A. Laib, P. Ruegsegger, The ability of three-dimensional structural indices to reflect mechanical aspects of trabecular bone, Bone 25(1) (1999) 55-60.

[46] T. Kawamoto, K. Kawamoto, Skeletal Development and Repair: Methods and Protocols, Humana Press2013.

[47] J.Y. Rho, L. Kuhn-Spearing, P. Zioupos, Mechanical properties and the hierarchical structure of bone, Medical engineering \& physics 20(2) (1998) 92-102.

[48] R.E. Guldberg, N.J. Caldwell, X.E. Guo, R.W. Goulet, S.J. Hollister, S.A. Goldstein, Mechanical stimulation of tissue repair in the hydraulic bone chamber, Journal of bone and 
mineral research : the official journal of the American Society for Bone and Mineral Research 12(8) (1997) 1295-302.

[49] I. Elgali, A. Turri, W. Xia, B. Norlindh, A. Johansson, C. Dahlin, P. Thomsen, O. Omar, Guided bone regeneration using resorbable membrane and different bone substitutes: Early histological and molecular events, Acta biomaterialia 29 (2016) 409-423.

[50] J.W. Hustedt, D.J. Blizzard, The controversy surrounding bone morphogenetic proteins in the spine: a review of current research, The Yale journal of biology and medicine 87(4) (2014) $549-61$.

[51] R. Fu, S. Selph, M. McDonagh, K. Peterson, A. Tiwari, R. Chou, M. Helfand, Effectiveness and harms of recombinant human bone morphogenetic protein- 2 in spine fusion: a systematic review and meta-analysis, Annals of internal medicine 158(12) (2013) 890-902.

[52] J. Dai, L. Li, C. Jiang, C. Wang, H. Chen, Y. Chai, Bone Morphogenetic Protein for the Healing of Tibial Fracture: A Meta-Analysis of Randomized Controlled Trials, PloS one 10(10) (2015) e0141670.

[53] M.A. Flierl, W.R. Smith, C. Mauffrey, K. Irgit, A.E. Williams, E. Ross, G. Peacher, D.J. Hak, P.F. Stahel, Outcomes and complication rates of different bone grafting modalities in long bone fracture nonunions: a retrospective cohort study in 182 patients, Journal of orthopaedic surgery and research 8 (2013) 33.

[54] J.E. Samorezov, E. Alsberg, Spatial regulation of controlled bioactive factor delivery for bone tissue engineering, Advanced drug delivery reviews (2014).

[55] R. Visser, P.M. Arrabal, J. Becerra, U. Rinas, M. Cifuentes, The effect of an rhBMP-2 absorbable collagen sponge-targeted system on bone formation in vivo, Biomaterials 30(11) (2009) 2032-7.

[56] T. Ratko, S. Belinson, D. Samson, C. Bonnel, M. Kathleen, N. Aronson, Bone Morphogenetic Protein: The State of the Evidence of On-Label and Off-Label Use, Blue Cross and Blue Shield Association Technology Evaluation Center Evidence-based Practice Center, 2010.

[57] N.J. Willett, M.T. Li, B.A. Uhrig, J.D. Boerckel, N. Huebsch, T.L. Lundgren, G.L. Warren, R.E. Guldberg, Attenuated human bone morphogenetic protein-2-mediated bone regeneration in a rat model of composite bone and muscle injury, Tissue engineering. Part C, Methods 19(4) (2013) 316-25.

[58] B.A. Uhrig, J.D. Boerckel, N.J. Willett, M.T. Li, N. Huebsch, R.E. Guldberg, Recovery from hind limb ischemia enhances rhBMP-2-mediated segmental bone defect repair in a rat composite injury model, Bone 55(2) (2013) 410-7.

[59] E.A. Wang, V. Rosen, J.S. D'Alessandro, M. Bauduy, P. Cordes, T. Harada, D.I. Israel, R.M. Hewick, K.M. Kerns, P. LaPan, D.P. Luxenberg, D. McQuaid, I.K. Moutsatsos, J. Nove, J.M. Wozney, Recombinant human bone morphogenetic protein induces bone formation, Proc Natl Acad Sci U S A 87(6) (1990) 2220-4.

[60] C.R. Dosier, B.A. Uhrig, N.J. Willett, L. Krishnan, M.T. Li, H.Y. Stevens, Z. Schwartz, B.D. Boyan, R.E. Guldberg, Effect of cell origin and timing of delivery for stem cell-based bone tissue engineering using biologically functionalized hydrogels, Tissue engineering. Part A 21(12) (2015) 156-65.

[61] D.J. Etherington, I.A. Silver, D.J. Restall, Resorption of insoluble, heterologous, fluorescein-collagen sponges in sensitized and non-sensitized rats, British journal of experimental pathology 60(6) (1979) 549-59. 
[62] M.A. James, Biological responses to materials, Annual Review of Materials Research 31(1) (2001) 81-110. 
A

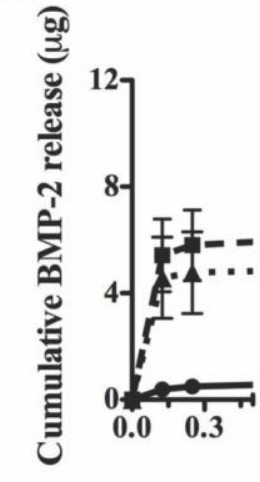

C
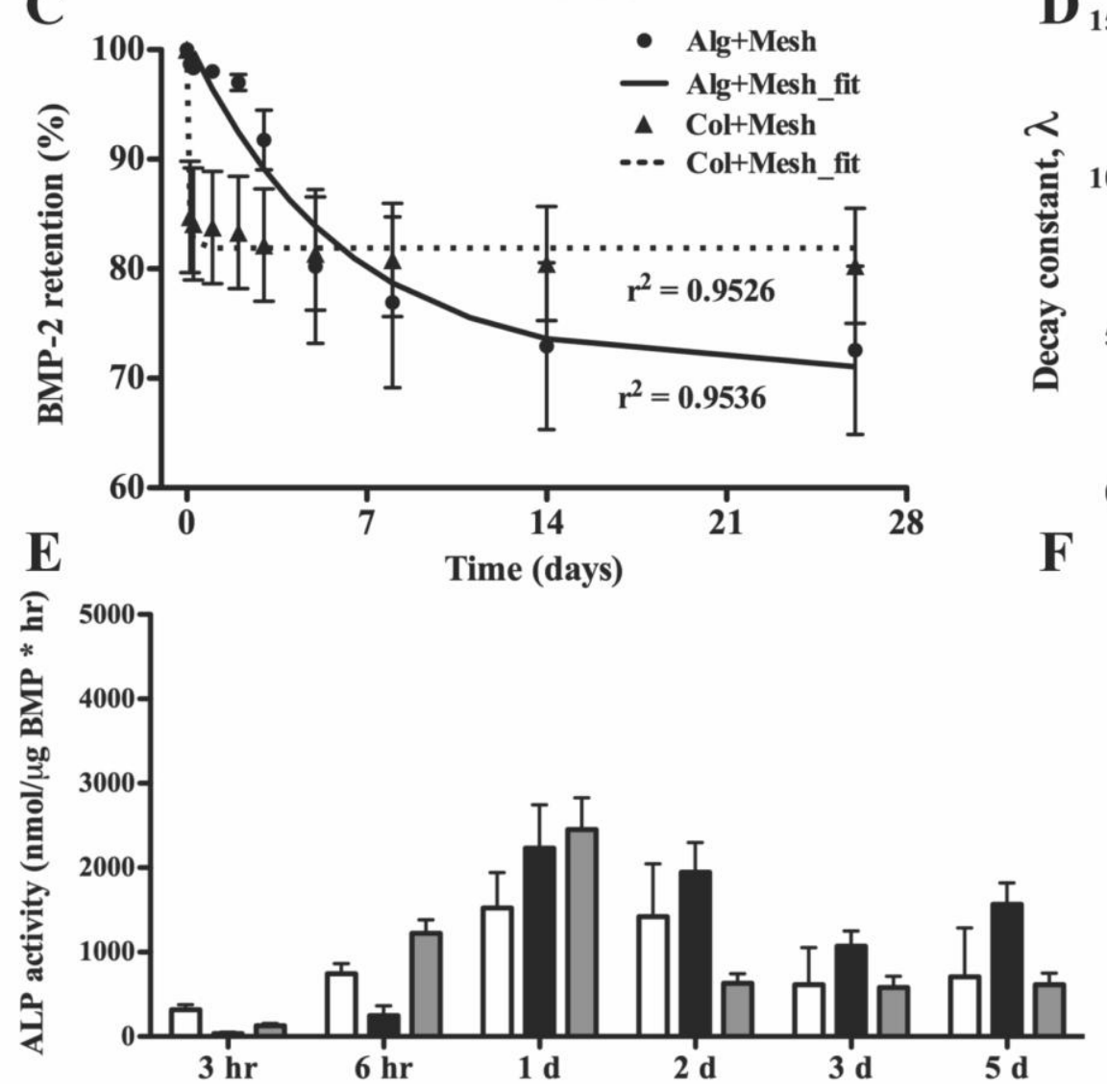
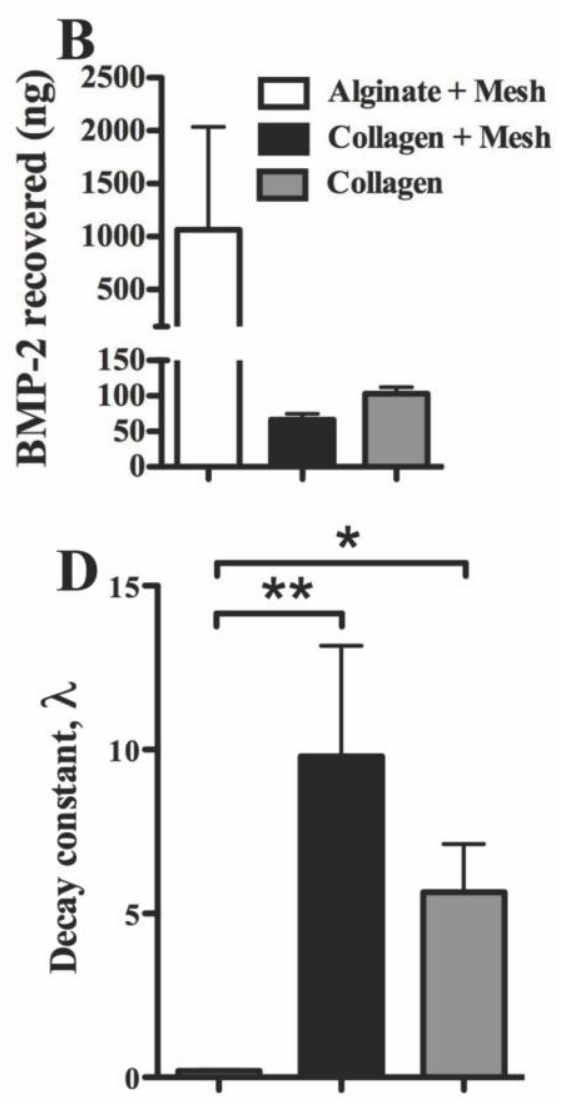

F

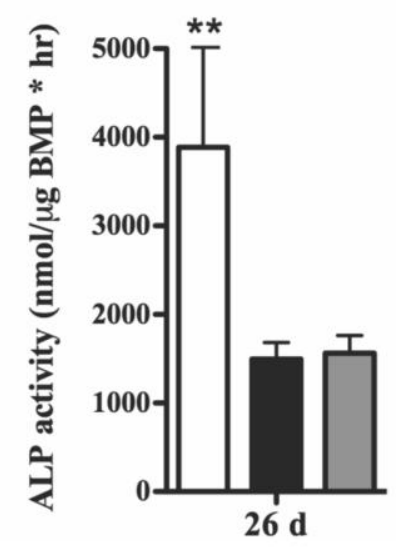



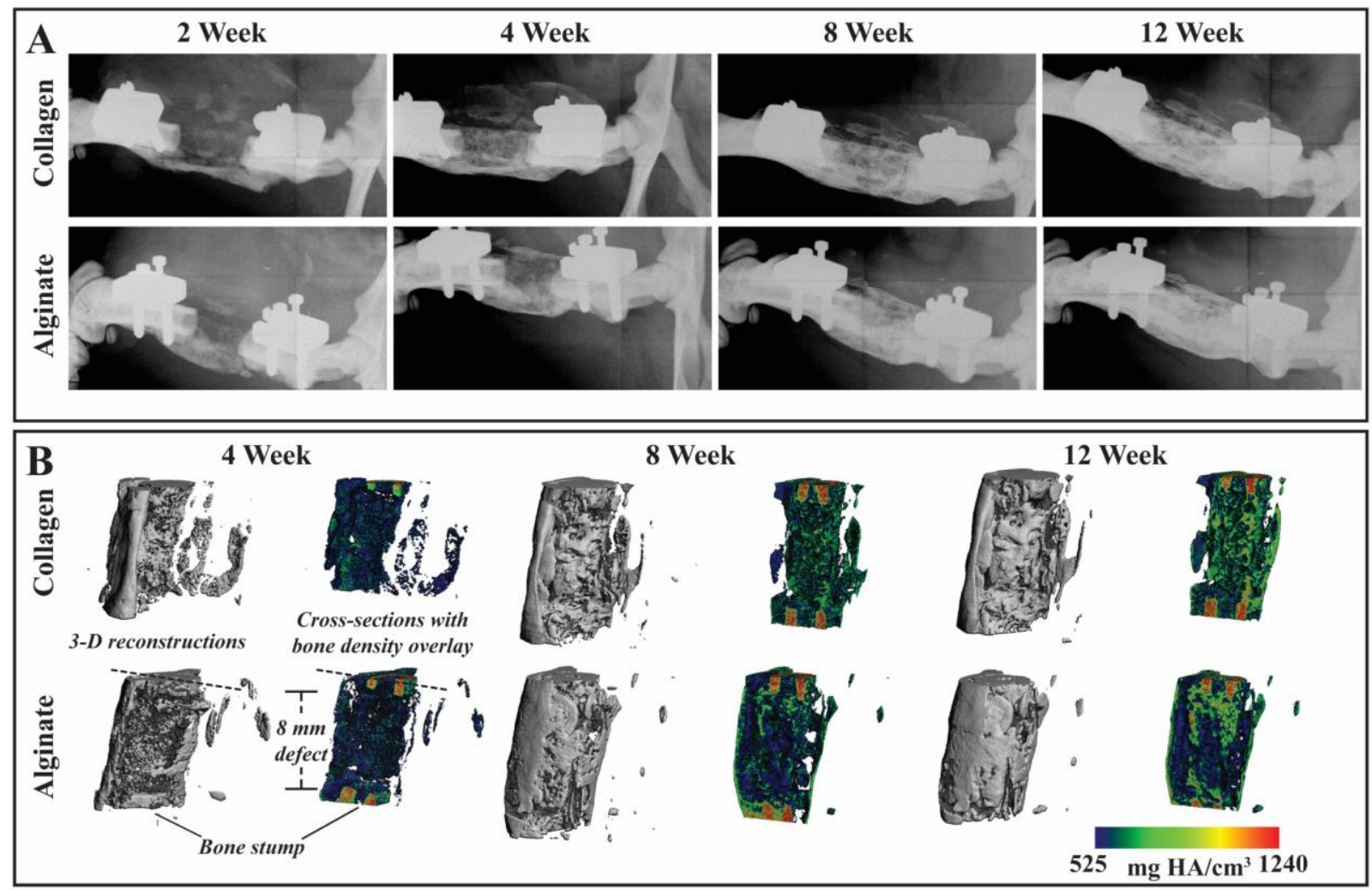
A

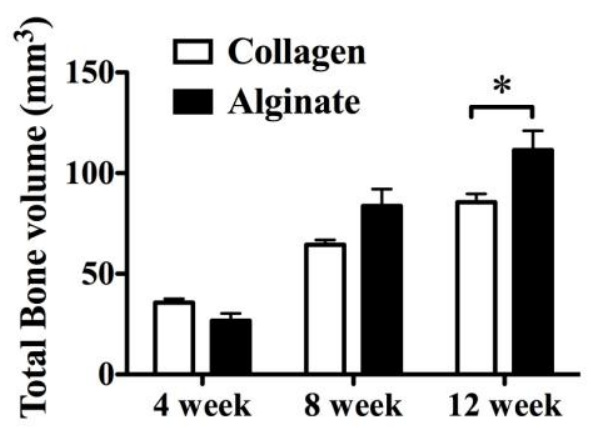

C

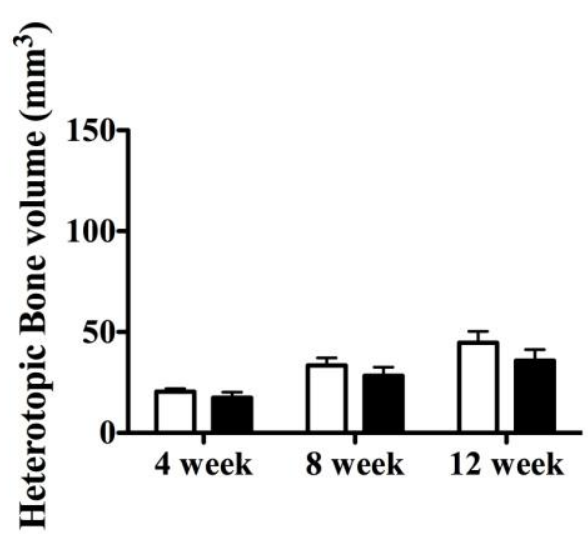

E

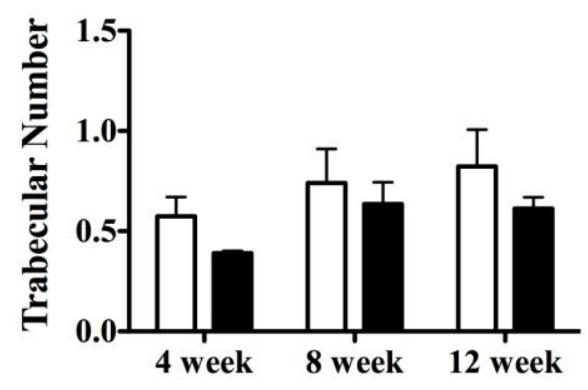

G

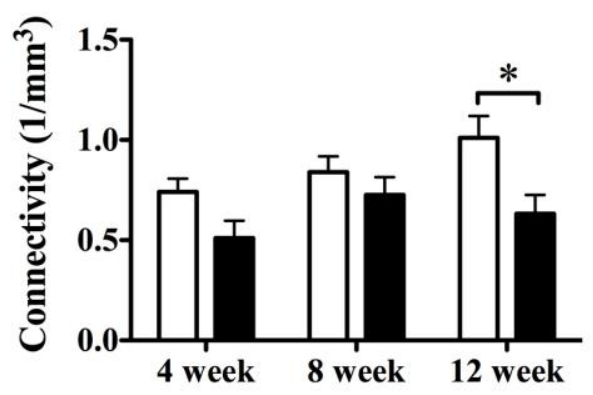

B

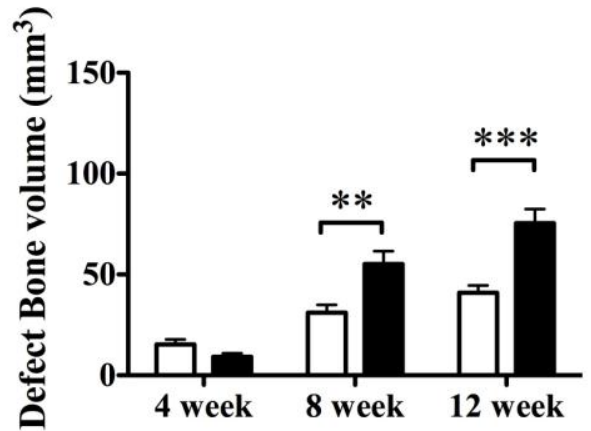

D

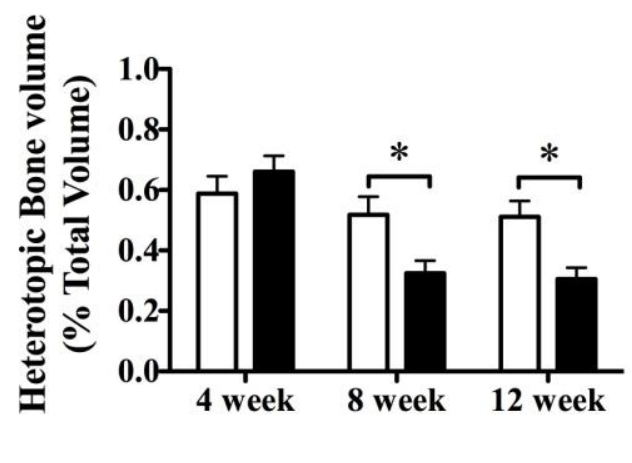

F

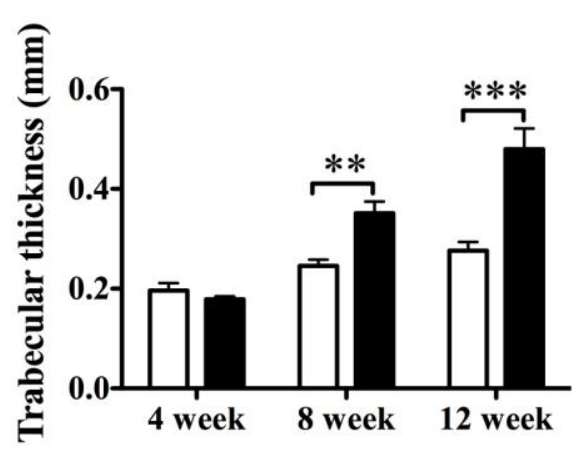

H

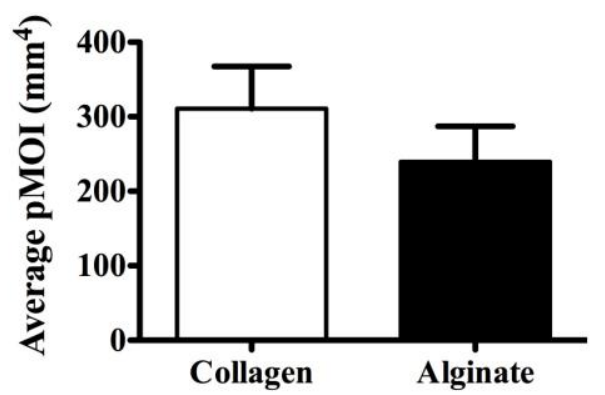




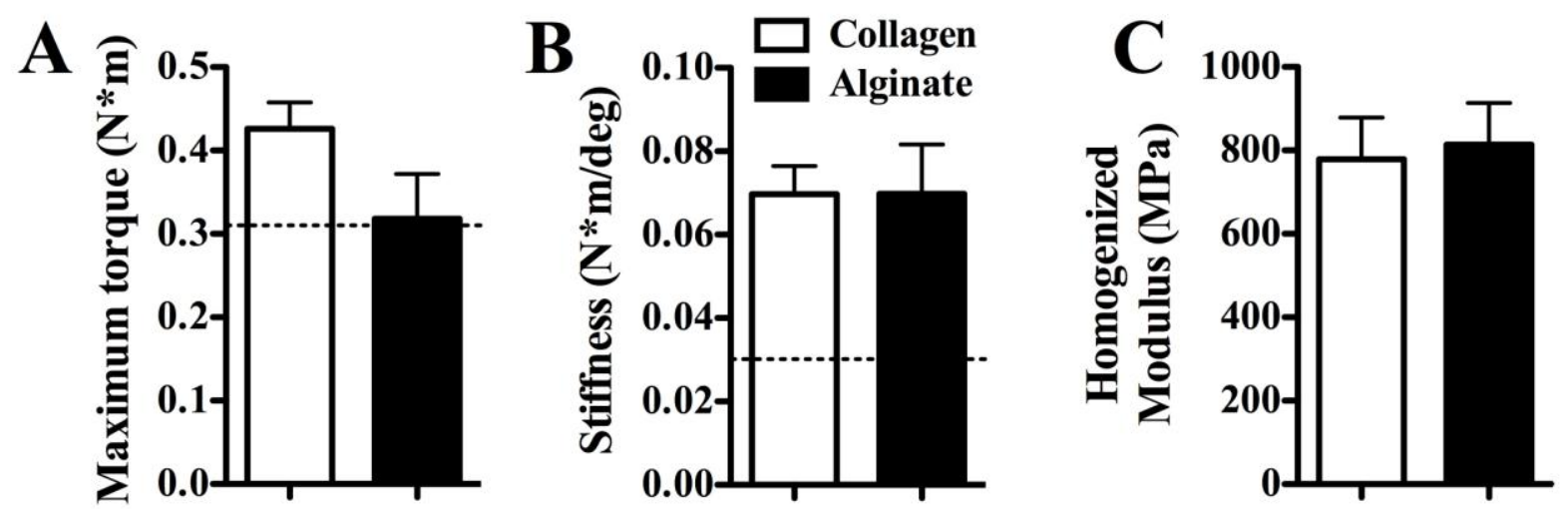

D

\section{Collagen}

Alginate
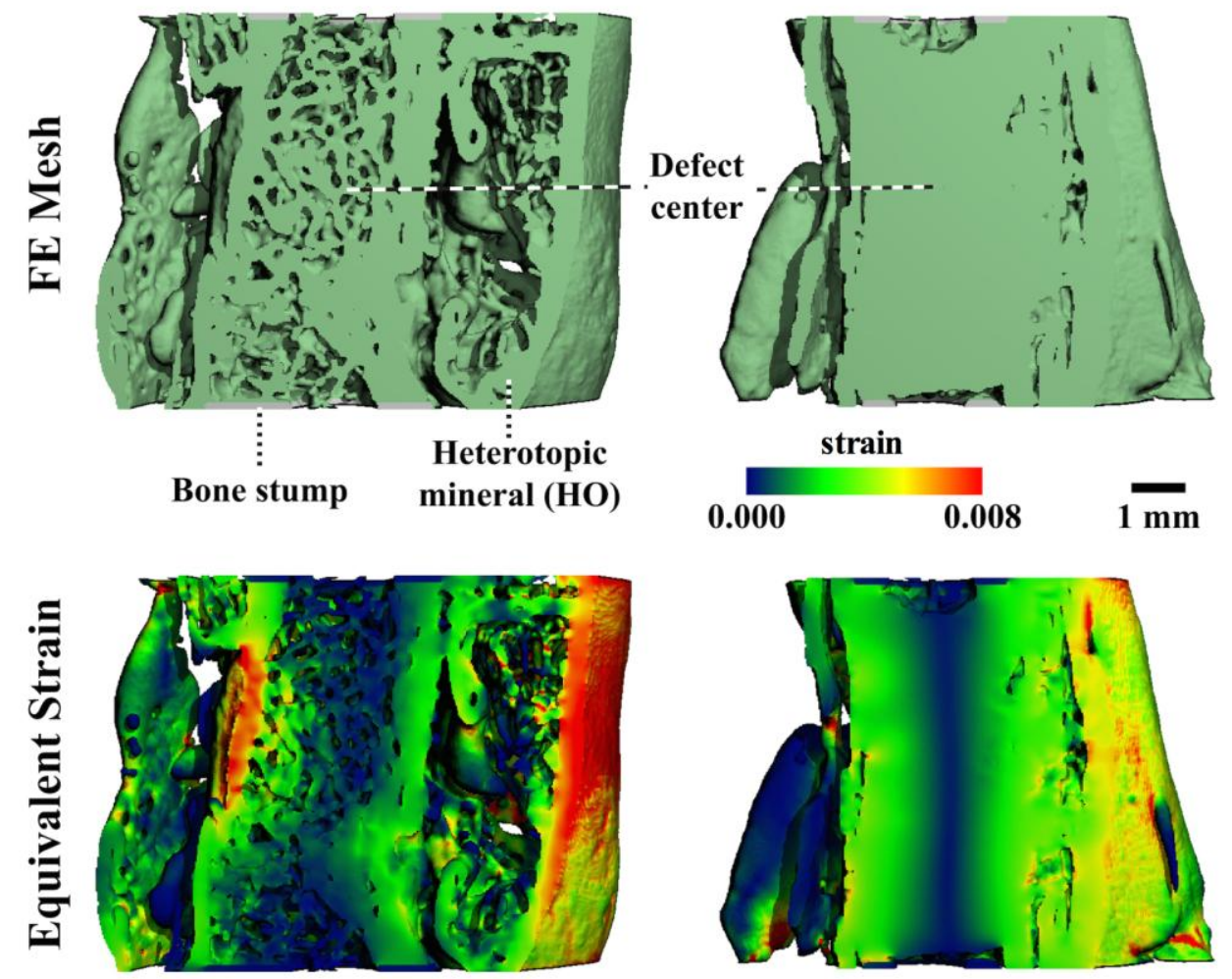


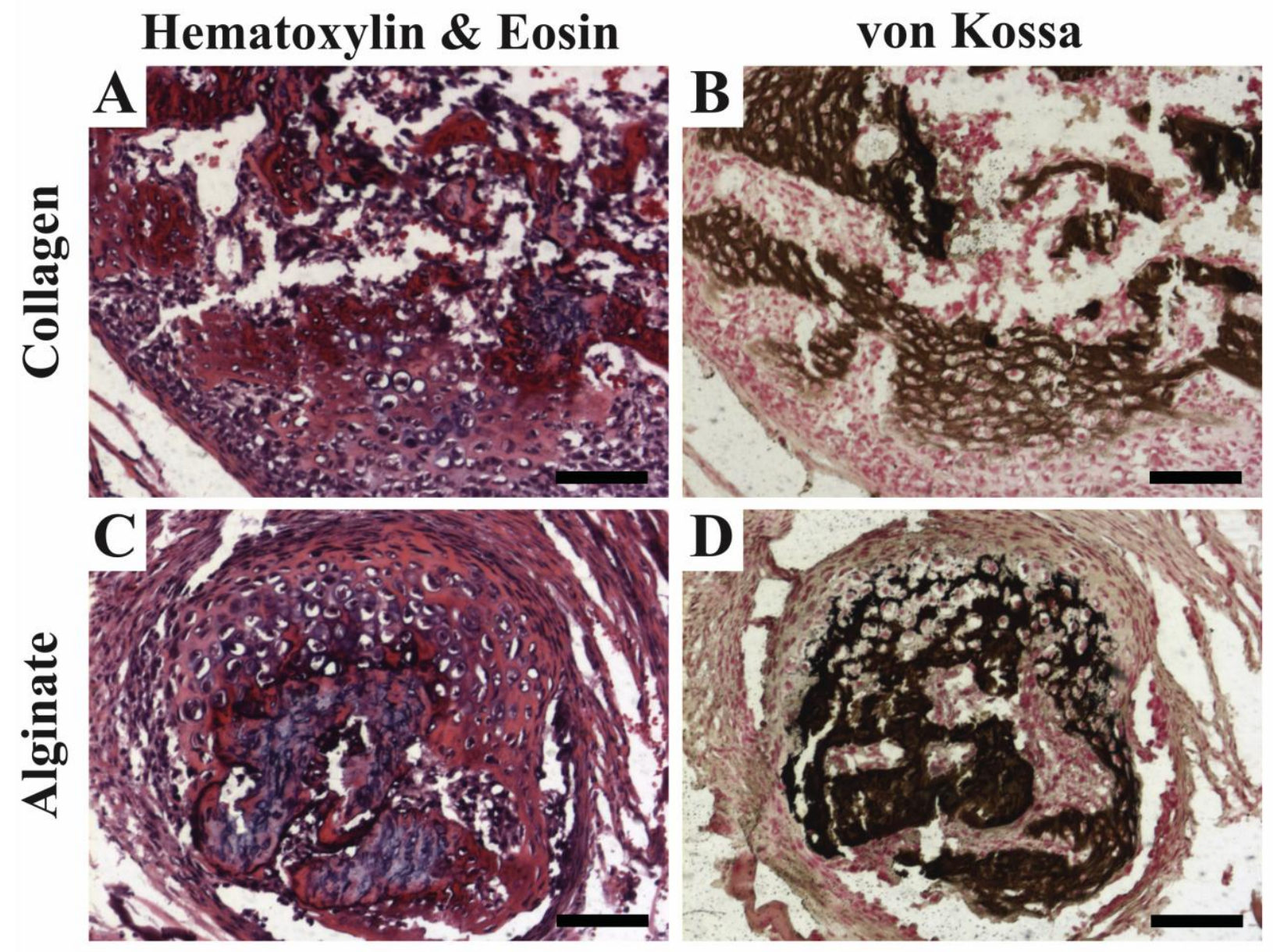



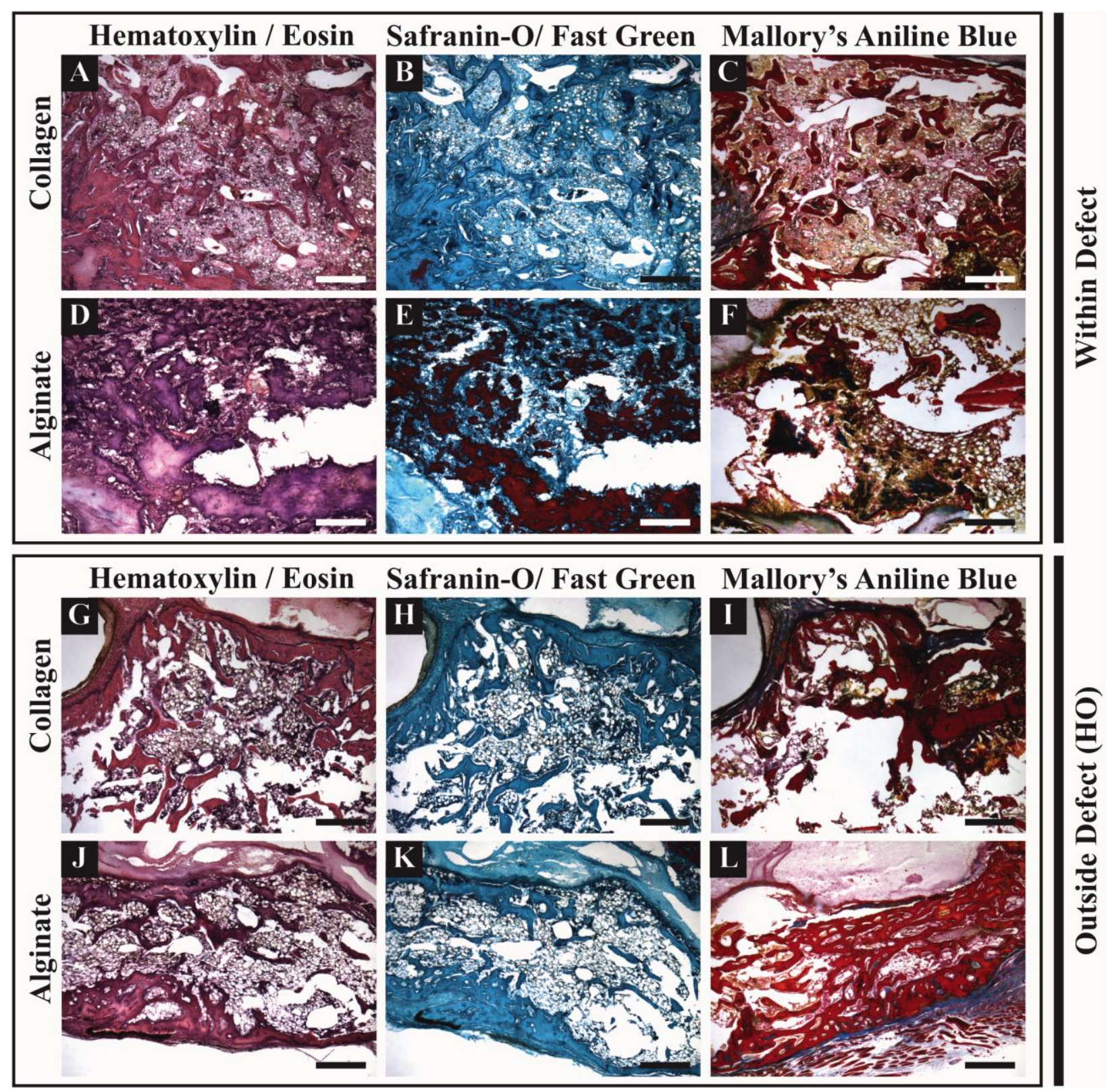


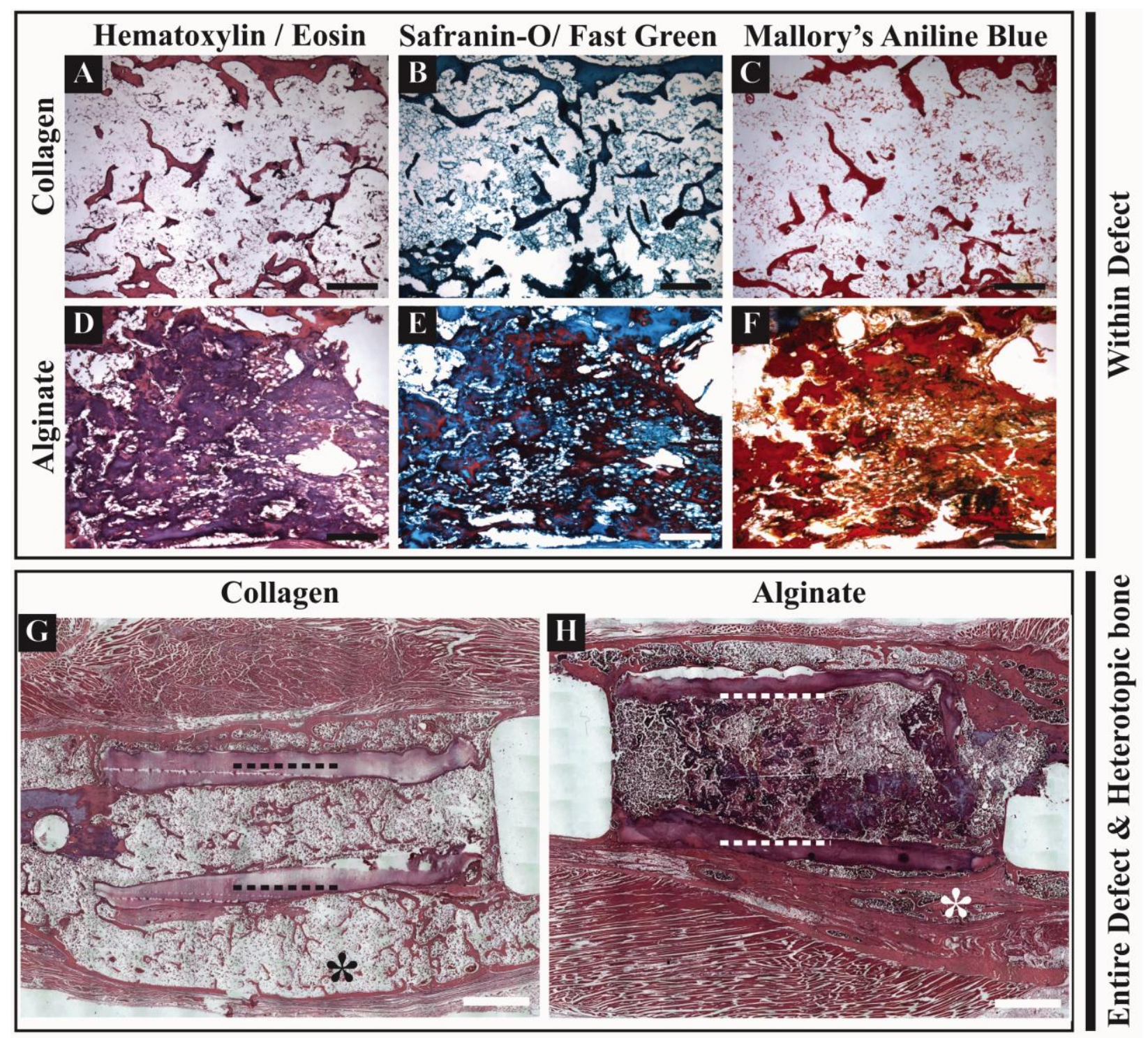



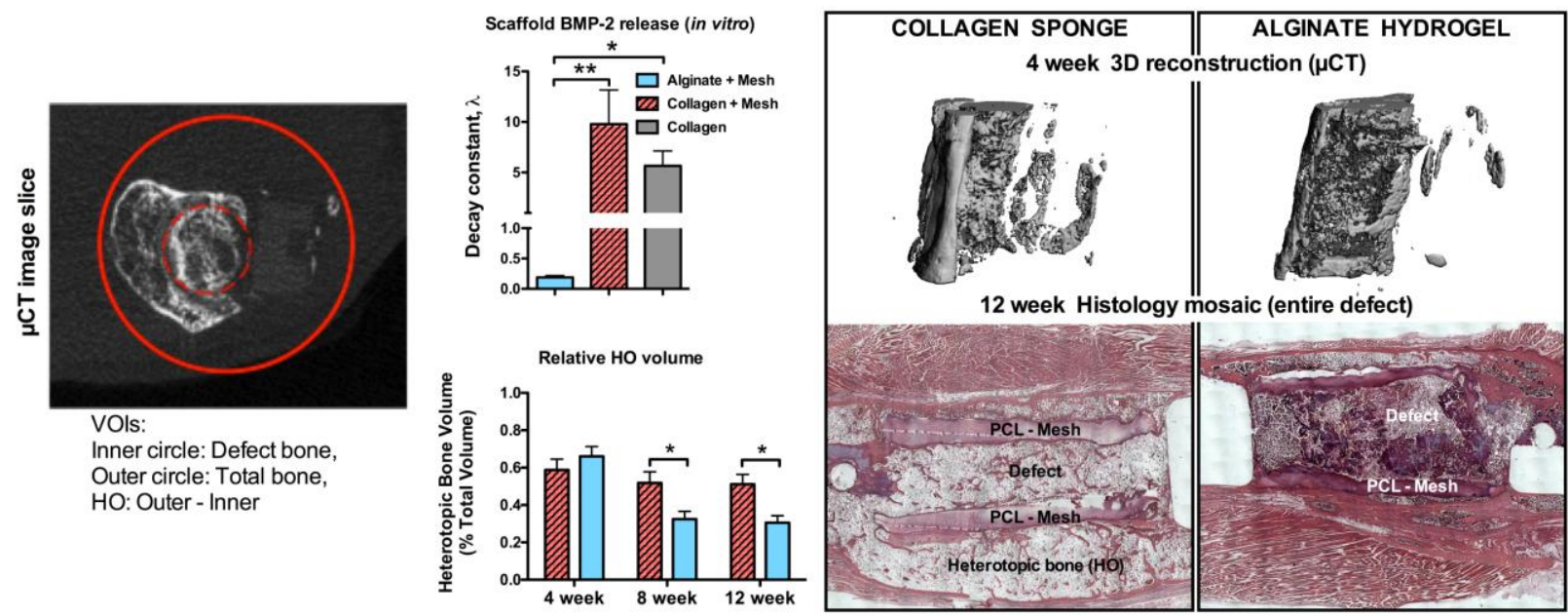